\title{
MR Imaging of the Hypermobile Lateral Meniscus of the Knee: A Case Report
}

\author{
Renuka Nair, Niraj Dubey \\ Department of Diagnostic Radiology, \\ Khoo Teck Puat Hospital, Singapore \\ Correspondence: \\ kunjukrisnan.renukanair@mohh.com.sg \\ Tel.: + 6591722666 \\ Fax.: + 6566023796 \\ Received: 17 December 2018 \\ Accepted: 6 June 2019
}

Key Words: Hypermobile Lateral Meniscus - Knee - Locking - Popliteomeniscal

Fascicle.

\begin{abstract}
Objective. The hypermobile lateral meniscus of the knee is a rarely described entity. In this case report we aim to draw attention to the clinical presentation and MR imaging findings of this pathology. Case Report. We review the clinical and imaging findings that led to the diagnosis of hypermobile lateral meniscus with transient subluxation causing intermittent locking, and which subsequently led to successful surgical treatment. Conclusion. Hypermobile lateral meniscus is rarely diagnosed prospectively on MR imaging. A better understanding of this uncommon condition will lead to prompt diagnosis and effective treatment, with a better outcome for the patient.
\end{abstract}

\section{Introduction}

The menisci are two C-shaped fibrocartilage structures in the knee joint (medial and lateral), which act as stabilizers and 'shockabsorbers. Both menisci are attached firmly to the tibial plateau by anterior and posterior roots. There are also peripheral attachments to these menisci, which are less extensive and laxer on the lateral meniscus (LM), making it more flexible and less prone to injuries compared to the medial meniscus (1).

In a seminal paper, correlated with an excellent anatomical demonstration, Resnick et al. (2) demonstrated the presence of ligamentous bands that anchor the posterior horn of the LM in its normal position so that it does not translate anteriorly upon knee flexion. They demonstrated the presence of popliteomeniscal fascicles (PMFs) that hold the posterior horn of the LM in place. The fascicles, namely the anteroinferior and the posterosuperior fascicles (Figure 1a-b), were shown to be fairly constant in most people, while an inconstant fascicle, the posteroinferior fascicle (Figure 1c) is seen rising more medially on the level of the attachment of the meniscofemoral Ligament of Wrisberg to the posterior horn. Injury to these fascicles or a congenital absence has been shown to be a major factor in excessive mobility of the posterior horn of the LM, a condition aptly named the 'hypermobile lateral meniscus' (3).

Hypermobile LM of the knee has not been widely described and is rarely diagnosed on prospective imaging. To our knowledge, based on a literature search, there are two other case reports of hypermobile lateral meniscus diagnosed on MRI and proven by arthroscopy $(4,5)$. In a case series of 13 patients with proven hypermobile lateral meniscus on arthroscopy (6), only one out of the 13 cases demonstrated an abnormality of the meniscus on MR imaging, where the knee was imaged in the locked position. 

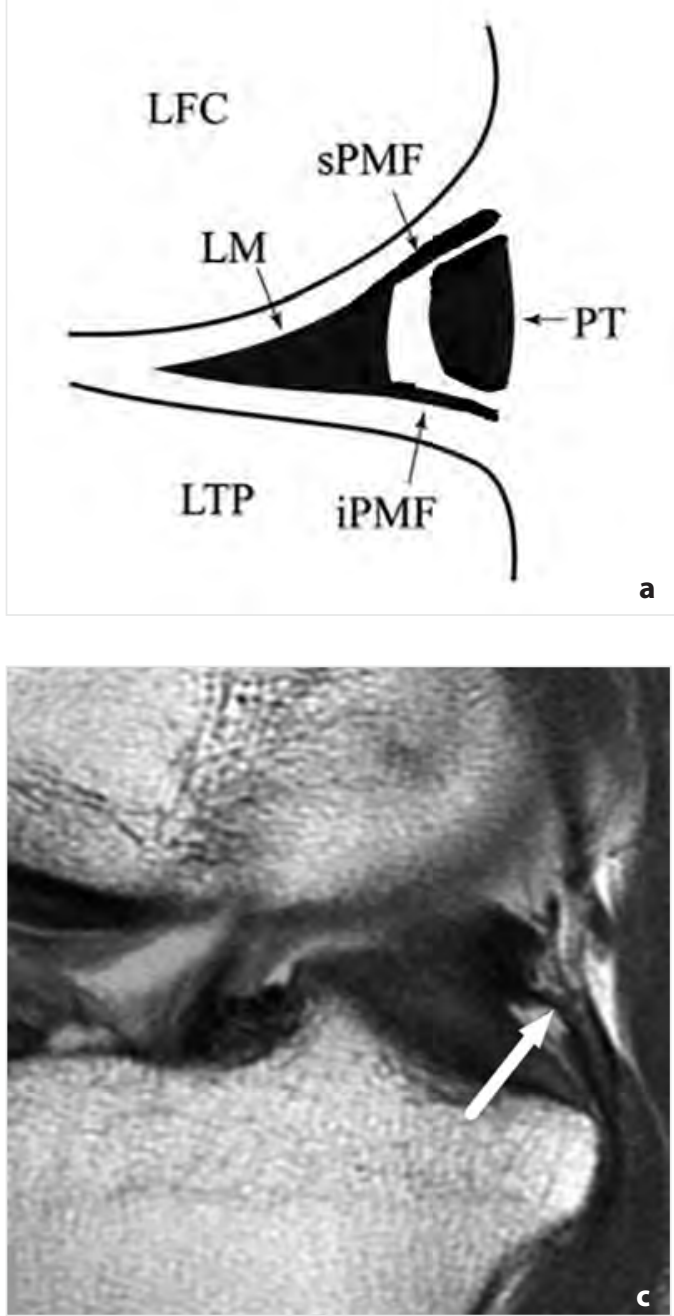

This abnormality was misdiagnosed as a bucket handle tear.

\section{Case Report}

An 18-year old girl presented with recurrent, intermittent episodes of painful locking of the left knee from 2010 up to the time of the last presentation in October 2015. In the interim, she had been examined in the orthopedic clinic with MR imaging of the affected knee in October 2013, which was reported as normal.

In the last presentation at the orthopedic clinic in October 2015, physical examina-

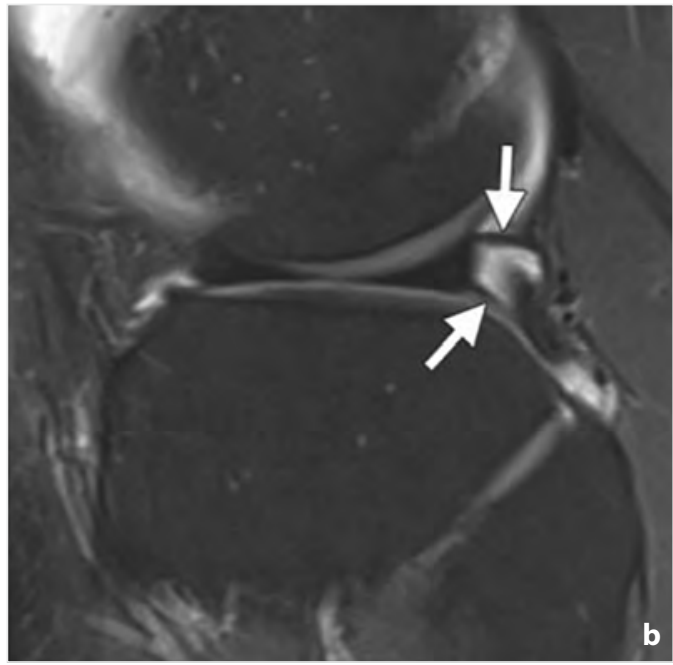

Figure 1.

(a) A drawing showing PMFs of knee joint. iPMF= Anteroinferior popliteomeniscal fascicle; LFC=Lateral femoral condyle; LM=Lateral meniscus; LTP=Lateral tibial plateau; PT=Popliteus tendon; sPMF=Posterosuperior popliteomeniscal fascicle.

(b) Sagittal PD FS image of a normal left knee. The sPMF and iPMF are clearly visualized (arrows).

(c) The posteroinferior popliteomeniscal fascicle (arrow) is not consistently visualized. It is seen near the root of the posterior horn and courses from the inferior margin of the meniscus posteroinferiorly to attach to a capsular extension of the popliteus tendon.

tion of the left knee revealed no swelling, redness or muscle wasting. There was limited range of motion of the knee joint. There was no patella apprehension but tenderness was felt over the medial patella region. Due to her unresolved symptoms, the patient was subsequently referred for a second MR imaging of the left knee.

In October 2015, MRI examination of the left knee of this patient was planned to be performed with the department's routine protocol. However, when the patient presented at our department on the day of the appointment, her knee was in a locked position, flexed at approximately $60 \mathrm{o}$. The mus- 
culoskeletal radiologist on duty that day decided to perform the MRI in this 'provoked' position. However, the patient could not fit into the GE Optima MR 430S-Extremity (GE Healthcare, Chicago, Illinois, United States) scanner or into the knee coil. As a result, the scan was performed on the $1.5 \mathrm{~T}$ MAGNETOM Avanto (Siemens Healthcare, Erlangen, Germany) scanner instead, with a body array coil used around the left knee. The sequences obtained were proton density (PD) weighted fat saturated (FS) images in axial, coronal and sagittal planes, coronal $\mathrm{T} 1$ weighted and $3 \mathrm{D}$ gradient echo in the sagittal plane. After the scan, the patient was able to extend her knee again. The MRI exam was then repeated in the neutral posi- tion utilizing our routine knee MRI protocol on the GE Optima MR 430S-Extremity (GE Healthcare, Chicago, Illinois, United States) scanner with a $145 \mathrm{~mm}$ knee coil. The sequences obtained were the PD weighted FS, in the axial, coronal and sagittal planes.

In the neutral position, the LM was essentially normal in appearance and position on all planes (Figure $2 \mathrm{a}-\mathrm{c}$ ). In the 'provoked' position, the posterior horn of the LM was anteriorly subluxated and interposed in the lateral tibio-femoral joint space (Figure 2df). The PMFs were absent (Figure 2f), which could be a result of a previous injury or a congenital absence. No meniscal tear was identified, but a LM flounce was noted. The final diagnosis was hypermobile posterior
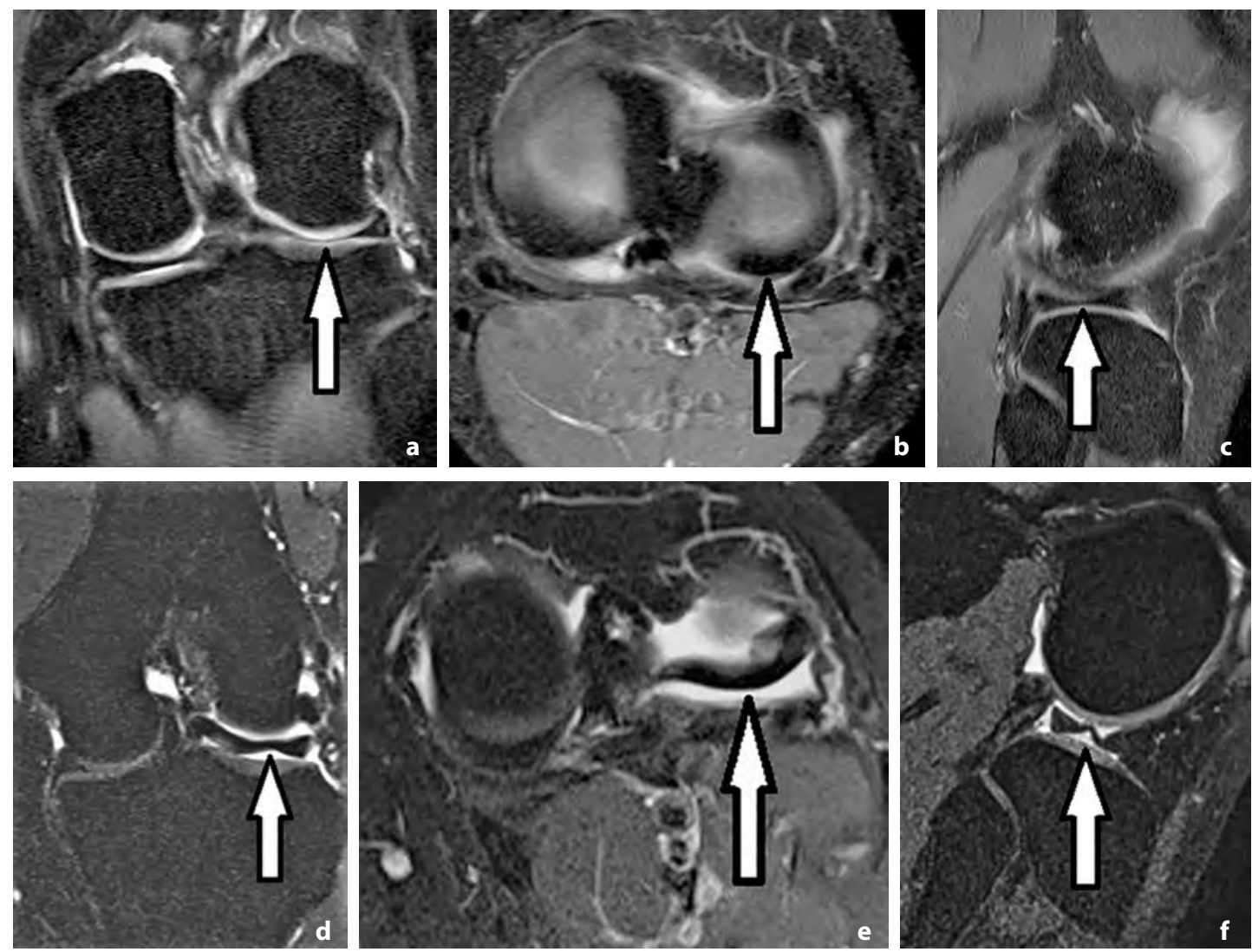

Figure 2. (a) Coronal (b) axial and (c) sagittal PD weighted FS MR images in the neutral position demonstrate a normal LM (arrows). (d) Coronal weighted PD FS MR image in the provoked position demonstrates a 'flounce' in the LM (arrow). (e) Axial PD FS MR image in provoked position demonstrates anterior subluxation of the posterior horn of the LM (arrow). (f) Sagittal gradient echo MR image demonstrates anterior subluxation of the posterior horn of the LM with a 'flounce' (arrow). This image also demonstrates the absence of the PMFs 
horn of the LM with transient subluxation causing intermittent locking.

After the diagnosis was made, the patient went on to have arthroscopic stabilization surgery. On examination under anesthesia, there was a positive pivot and Lachman test. Arthroscopy revealed an intact anterior cruciate ligament (ACL) and posterior cruciate ligament (PCL). The medial meniscus was intact. There was hypermobility and subluxability of the posterior horn of the LM. No tear was detected. Stabilization of the posterior horn was performed with fixation of the posterior horn of the LM to the posterior capsule using Fast-Fix 360 meniscal repair device (Smith \& Nephew, London United Kingdom), which is a device normally used for a minimally invasive repair of posterior and middle third tears of the meniscus using a vertical mattress suture. The patient was discharged in good health the following day. Her symptoms have resolved since then.

\section{Discussion}

The hypermobile LM refers to the condition where the LM loses its posterior anchoring i.e. the PMFs becomes very lax and the LM subluxates, mainly with flexion. The patient presents with a range of symptoms in terms of severity, most commonly pain and locking of the knee joint, in the absence of a meniscal tear or discoid meniscus (7). PMFs are structures that are not routinely assessed by most radiologists, hence the low incidence of the diagnosis of the hypermobile lateral meniscus of the knee on MR imaging. The diagnosis is usually made by arthroscopy (8).

In our case, the initial MR imaging performed in October 2013 with the routine protocol i.e. with the knee in the neutral position, was reported as normal. In retrospect, the anteroinferior and the posterosuperior PMFs were not visualized while the posteroinferior PMF was partly seen. On the subsequent MR imaging performed in October
2015, imaging the left knee in the provoked, symptomatic flexed position, it was possible to demonstrate the anterior subluxation of the posterior horn of the LM and the condition of the absent PMFs became more apparent. In the same setting, with the knee in the neutral position, none of the three PMFs are seen. A helpful pointer in this regard is the presence of an LM flounce, which is often considered normal in the medial meniscus, but its presence in the LM should prompt a search for the condition of the absent PMFs (9), in the absence of a meniscal tear. In our patient, the flounce of the LM was detected in the provoked position.

\section{Conclusion}

Hypermobile LM is a poorly recognized entity which is rarely diagnosed prospectively on MR imaging. Increased awareness of the clinical presentation, and the anatomy and pathology of the PMFs, will lead to accurate and prompt diagnosis with effective treatment and better outcome for the patient. Pointers that could help radiologists in diagnosis are looking for the presence of a lateral meniscal 'flounce' and performing MR imaging of the knee in the provoked position, whenever feasible, in a patient with typical symptoms.

\section{What is Already Known on this Topic}

The posterior ligamentous bands, called the popliteomeniscal fascicles, anchor the lateral meniscus to its normal position, preventing it from anterior subluxation during flexion. The absence of these bands, either due to trauma or congenitally, could lead to an entity called 'hypermobile lateral meniscus'. The patient commonly presents with knee pain and locking. This entity is not widely described and commonly missed on prospective imaging, if not scrutinized carefully.

\section{What this Case Report Adds}

We present a case of the hypermobile lateral meniscus which was not diagnosed on prospective imaging in 2013. On the repeat MR imaging of the affected knee in October 2015, the pathology was demonstrated when the knee was in the flexed and locked position. A lateral meniscal 'flounce' was also demonstrated. These could be pointers to help radiologists in seeking out this pathology when presented with a similar case. 
Authors' Contributions: Conception and design: ND; Acquisition, analysis and interpretation of data: ND and RN; Drafting the article: RN; Revising it critically for important intellectual content: ND; Approved final version of the manuscript: ND and RN.

Conflicts of Interest: The authors declare that they have no conflicts of interest.

\section{References}

1. Thornton DD, Rubin DA. Magnetic resonance imaging of the knee menisci. Semin Roentgenol. 2000;35(3):217-30.

2. Peduto AJ, Nguyen A, Trudell DJ, Resnick DL. Popliteomeniscal fascicles: anatomic considerations using MR arthrography in cadavers. AJR Am J Roentgenol. 2008;190(2):442-8.

3. MRI Web Clinic [homepage on the internet]. Mark HA. Hypermobile lateral meniscus. [citation date; February 2015]. Available from: http://radsource. us/hypermobile-lateral-meniscus/\#fn-6197-18.

4. George M, Wall EJ. Locked knee caused by meniscal subluxation: magnetic resonance imaging and arthroscopic verification. Arthroscopy. 2003; 19(8):885-8.

5. Lyle NJ, Sampson MA, Barrett DS. MRI of intermittent meniscal dislocation in the knee. Br J Radiol. 2009;82(977):374-9.

6. Oyster M, Smerek JP, Kaeding CC. Paper 272: Hypermobile Posterior Horn Lateral Meniscus. Arthroscopy. 2012;28(9 Suppl):e499.

7. Van Steyn MO, Mariscalco MW, Pedroza AD, Smerek J, Kaeding CC, Flanigan DC. The hypermobile lateral meniscus: a retrospective review of presentation, imaging, treatment, and results. Knee Surg Sports Traumatol Arthrosc. 2016;24(5):1555-9.

8. Suganuma J, Mochizuki R, Inoue Y, Yamabe E, Ueda Y, Kanauchi T. Magnetic resonance imaging and arthroscopic findings of the popliteomeniscal fascicles with and without recurrent subluxation of the lateral meniscus. Arthroscopy. 2012;28(4):507-16.

9. Yu JS, Cosgarea AJ, Kaeding CC, Wilson D. Meniscal flounce MR imaging. Radiology. 1997;203(2): 513-5. 